\title{
Development of a Cost-Effective PC-Based Motion Control System
}

Federal Manufacturing \& Technologies

D. L. Hollar

KCP-613-6037

Published January 1998

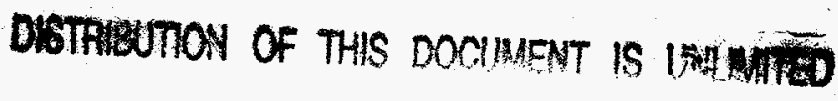

Final Report

Approved for public release; distribution is unlimited.

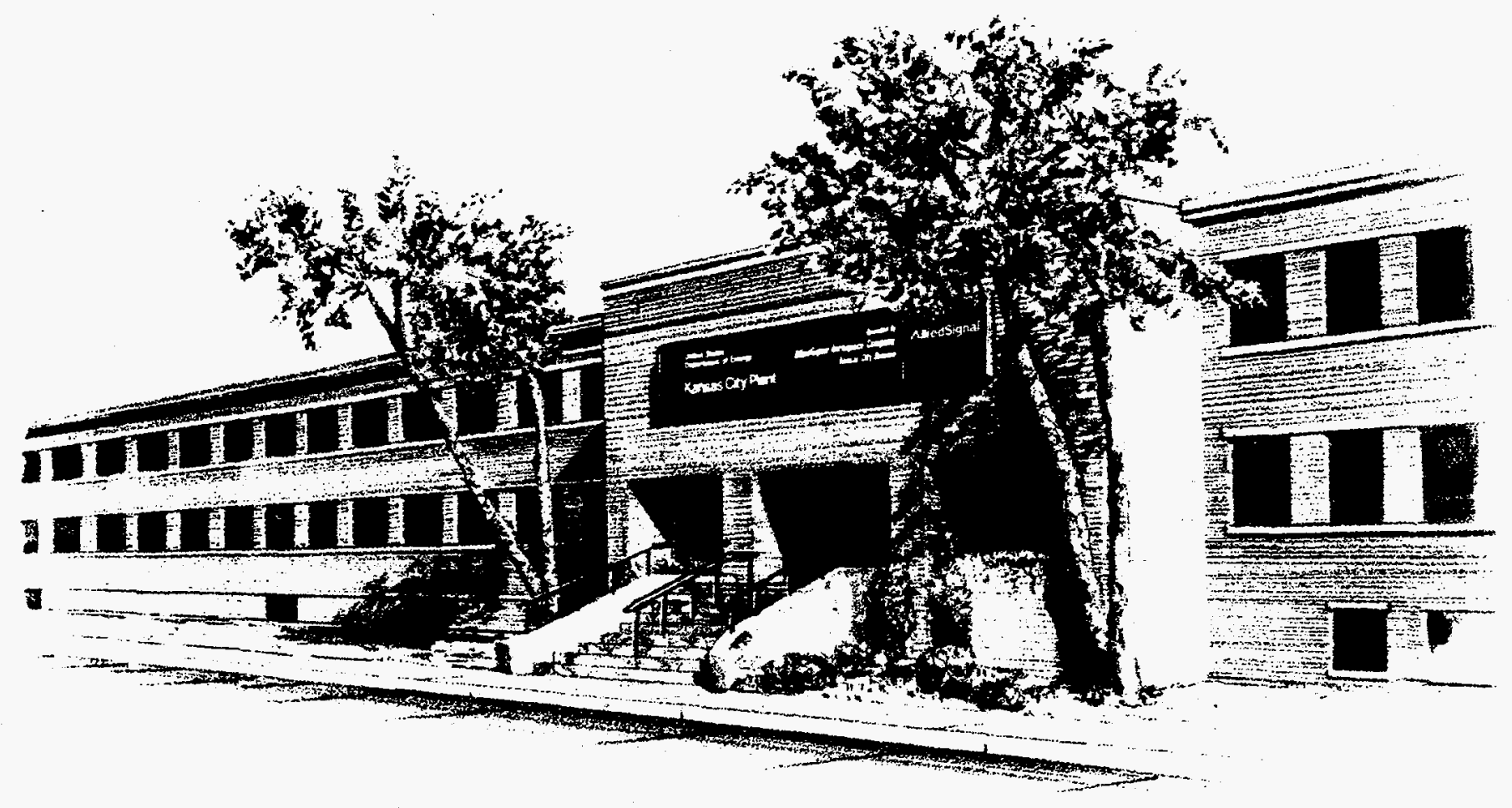

Prepared Under Contract Number DE-ACO4-76-DP00613 for the United States Department of Energy 


\section{DISCLAIMER}

This report was prepared as an account of work sponsored by an agency of the United States Government. Neither the United States Government nor any agency thereof, nor any of their employees, make any warranty, express or implied, or assumes any legal liability or responsibility for the accuracy, completeness, or usefulness of any information, apparatus, product, or process disclosed, or represents that its use would not infringe privately owned rights. Reference herein to any specific commercial product, process, or service by trade name, trademark, manufacturer, or otherwise does not necessarily constitute or imply its endorsement, recommendation, or favoring by the United States Government or any agency thereof. The views and opinions of authors expressed herein do not necessarily state or reflect those of the United States Government or any agency thereof. 


\section{DISCLAIMER}

Portions of this document may be illegible electronic image products. Images are produced from the best available original document. 
KCP-613-6037

Distribution Category UC-706

Approved for public release; distribution is unlimited.

DEVELOPMENT OF A COST-EFFECTIVE

PC-BASED MOTION CONTROL SYSTEM

D. L. Hollar

Published January 1998

Final Report

D. L. Hollar, Project Leader

Project Team:

R. H. Cottrell

A. W. Robbins 


\section{Contents}

Section

Page

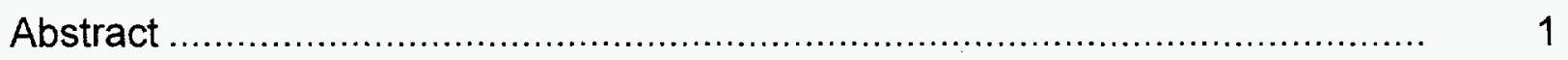

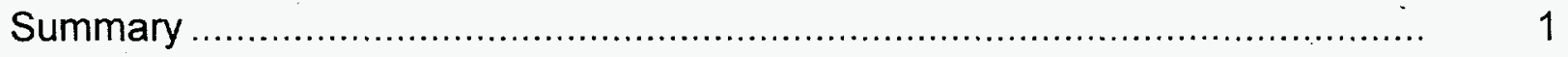

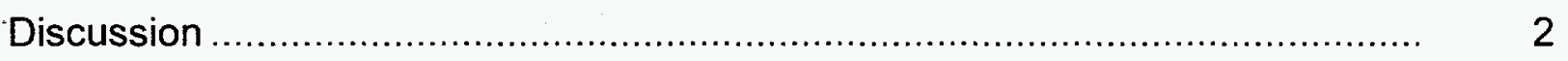

Scope and Purpose............................................................. 2

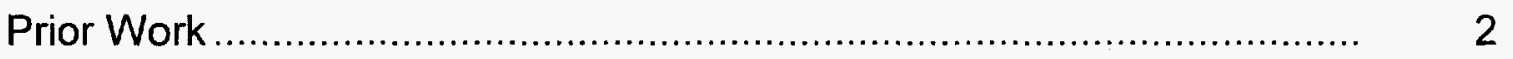

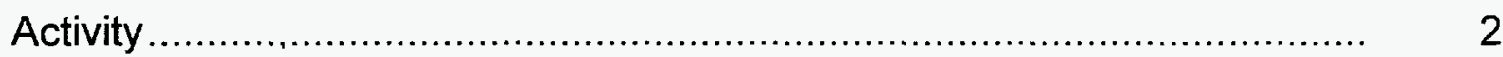

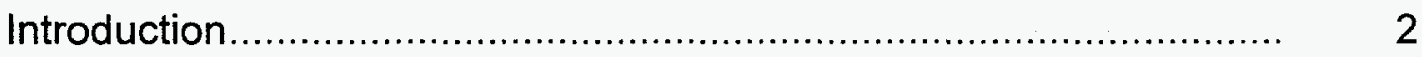

PC Motion System Requirements ....................................... 3

Proposal Requirements ................................................ 4

Software Development ................................................... 4

PC Motion System Properties ......................................... $\quad 5$

Advantages of the New Windows-Based Motion System................. 8

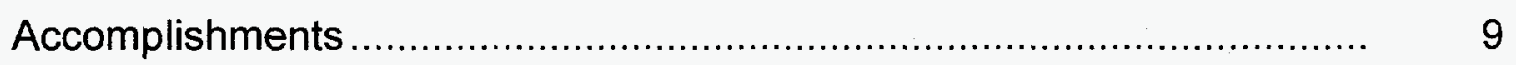




\section{Illustrations}

Figure Page

1 Edit Páge Provides Path Programming and Editing ........................... 5

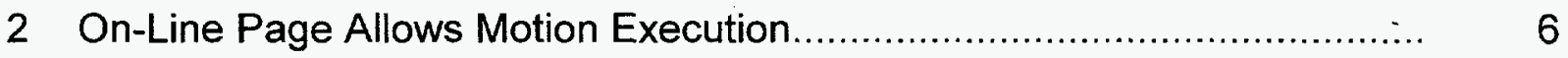

3 The Jog Window Allows Specific X-Y or Rotary Movements $\ldots \ldots \ldots \ldots \ldots \ldots \ldots . . \ldots$

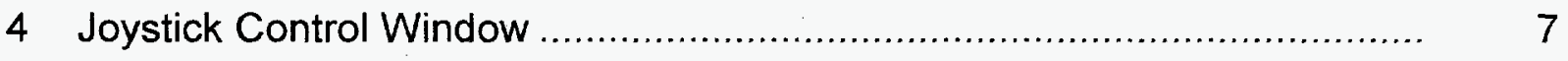

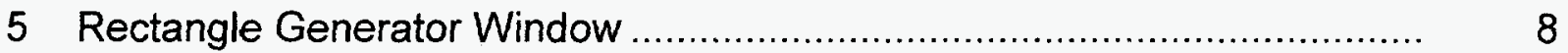




\section{Abstract}

A cost-effective PC-based motion control system was developed and evaluated for use on a laser welding system. The motion system is capable of $X-Y$ simultaneous contouring and provides a rotary axis of motion also. The system motion paths can be specified in either Relative or Absolute motion. The PC controls all of the laser power supply and shutter I/O operations. All of the motion programming and operator interfacing is via the Windows $\otimes 95$ operating system.

\section{Summary}

The goal of this project was to develop a cost-effective X-Y motion system suitable for use on an existing YAG laser welder. Generally a commercially available machine tool computer numerical control (CNC) motion system would be used for this type of motion application. However, the CNC systems are costly $(\$ 35 \mathrm{~K}-\$ 50 \mathrm{~K})$ and require considerable programming training to create motion paths.

The laser welding system on this project currently has a 12" by 12 " X-Y table with manual joystick positioning capability. The tables are driven by low-cost stepper motors and the associated electronic drivers. Stepper motor technology and the drive electronics have recently advanced to where they nearly match the performance of the more expensive DC servomotor/encoder and CNC combination found in the commercial systems. By using the computing power of a modern inexpensive personal computer (PC) and the stepper motor interface electronics, a cost-effective motion system can be assembled.

The only shortcoming of the stepper motor application is the specialized programming knowledge required to program the stepper motor drives. It was thought that the ideal motion system should use a Windows $\otimes$-based programming protocol since most people are familiar with Windows-type applications. A software vendor experienced in stepper motor systems was contracted to develop a Windows-based operator interface.

The marriage of the PC, stepper motor technology, and custom software has produced a very user-friendly high performance motion system at a reasonable cost. It provides simultaneous $X-Y$ contouring, input/output $(I / O)$ controls, and rotary positioning. While the initial cost to develop the software $(\$ 32 \mathrm{~K})$ seems expensive, it is a one-time investment. Federal Manufacturing \& Technologies (FM\&T) owns the software and can load it on as many systems as needed at minimal additional cost. All that will be required to create additional motion systems is the purchase of the hardware, typically $\$ 15 \mathrm{~K}$ for a PC, $\mathrm{X}-\mathrm{Y}$ tables, motors, and drive electronics. In addition, the motion system is not limited to laser weld systems. The motion system could be used on nearly any automated motion application. 


\section{Discussion}

\section{Scope and Purpose}

The purpose of this project was to develop a cost-effective $X-Y$ motion control system suitable for use on an existing laser welder. Commercially available $X-Y$ motion systems consist of a dedicated numerical controller and a motion table for each axis. Generally these systems cost in the range of $\$ 35 \mathrm{~K}$ to $\$ 50 \mathrm{~K}$ for 12 inches of $X-Y$ travel. Since most of the commercial motion systems are designed for machine tool numerical control applications, they provide very good accuracy and performance. However, the motion requirements for laser welding are generally less demanding than a machine tool operation.

The scope of this project included the development and evaluation of a motion system using a PC, 12-inch X-Y tables, and a combination of commercial software with some custom-developed software. Recent developments in PCs, PC accessory hardware, software, and motion table drive systems have made a cost-effective motion system available for less than $\$ 15 \mathrm{~K}$. The goal of this project was to develop and investigate the performance of one of these systems.

\section{Prior Work}

The development of laser soldering technology on an earlier project also required the development of a specialized motion system. The hardware and software encountered on the project are similar to the hardware and software requirements for this project. Experience gained by the software vendor on the earlier project was leveraged to reduce the time and cost associated with the development of the subject laser welding motion system.

\section{Activity}

\section{Introduction}

In the past, various forms of motion systems have been utilized for development welding systems. These ranged from simple single-axis positioning to commercial NC-based 3-axis controllers. The laser welder is currently equipped with a joystickcontrolled, 12-inch X-Y table/stepper motor system. Implementation of these motion systems provided knowledge and experience with the evolution of stepper motors and stepper motor drives. Stepper motors are an economical alternative to the more expensive DC servomotor/encoder combination commonly used for motion table drives. Advances in stepper motor technology have made them very competitive with the DC servomotor systems, especially for less demanding positioning applications. 
Manufacturers of stepper motor products have also developed a large variety of electronic motor drive systems, including interface circuit boards which can be installed in a PC. Combining the computing power of a PC with one these interface boards and an electronic drive creates an economical and versatile motion system. The interface boards allow several channels of $\mathrm{I} / \mathrm{O}$ controls in addition to providing interfacing with the motor drives. The stepper motor vendors have developed companion software to go with their electronic drive and $\mathrm{l} / \mathrm{O}$ systems.

While these PC-based motion systems provide excellent motion control capability, the companion software required to program motion commands requires programming knowledge specifically for that product line. Early attempts to use the software revealed a steep learning curve to efficiently create motion paths. The software is quite powerful but is generally not user friendly. As a result, a programming contractor is usually employed to develop and maintain specific motion programs.

\section{PC-Motion System Requirements}

A cost-effective motion system was the primary goal of this project; however, the other major interest was to provide a user-friendly system. Choosing the stepper motor, electronic drive, and PC control hardware immediately resulted in much lower motion system hardware costs. However, using the companion software with its complexities would not provide the desired flexible user-friendly programming. Therefore, custom programming software will be required to achieve a user-friendly system.

A secondary requirement of this project was that the system be developed with the potential that it could to be used on other automation tasks. Some welding operations may only require one axis of motion and a few $1 / O$ controls, while other manufacturing operations could potentially benefit from a similar form of automation. The system design philosophy required that it could be used on nearly any positioning task.

A meeting was conducted with a software programming vendor to establish what type of operator/system interface could be developed. As noted in the Prior Work section, this vendor was selected because of familiarity with the existing Compumotor $\circledast$ stepper motor hardware already installed in the laser welder. Based on the information gained from the meeting and inputs solicited from current laser system users, system requirements were complied. These requirements were drafted in a "request for proposal" and submitted to the vendor for quotation. 


\section{Proposal Requirements}

All production laser weld systems at Federal Manufacturing \& Technologies (FM\&T) use conventional CNC motion systems. Most engineers and machine operators are familiar, to varying degrees, with the somewhat cryptic code normally encountered in CNC programming. Therefore, part of the user friendliness of the new system should overcome the CNC programming complexities, although it was thought to be desirable to keep some of the logical CNC operations and motion path conventions to reduce the confusion when moving from the CNC to the PC system.

It was proposed that the new motion system use a Windows environment considering the general population's widespread knowledge of Windows applications. Therefore, the first requirement specified that the PC use the Windows environment for all operator/motion system interfacing. That would allow both keyboard and mouse inputs. The vendor indicated that several Windows construction software packages were available. The motion paths should be capable of circular interpolation, i.e., continuous $X-Y$ contour moves. Motion movement should be controlled in either Relative or Absolute movement. All laser power supply and shutter I/Os should be controllable from the PC. A graphic display of the motion path should be presented in a window on the screen, along with programming functions. The graphic display is usually an option only available in high end CNC systems.

\section{Software Development}

The software vendor and the FM\&T project team reviewed the contract requirements and agreed on the system attributes. The contract was awarded at a cost of $\$ 32 \mathrm{~K}$. While the initial cost to develop the software seems expensive, it is a one-time investment. FM\&T owns the software and can load it on as many systems as needed at minimal additional cost. All that will be required to create additional motion systems is the purchase of the hardware.

The vendor completed a preliminary version of the software and installed it on the FM\&T PC platform. The software was constructed with Microsoft Visual Basic $\otimes$ and uses the Windows 95 operating system. The team members exercised the software and found some flaws as well as discovering some ideas that would be desirable additions. The vendor was very cooperative by incorporating the new features even though these features were not defined in the original proposal. After correcting the flaws and incorporating the new features, a final version of the software package was completed and approved. The delivered package also included two copies of an instruction manual, all system documentation, and a license for all of the purchased construction software. 


\section{PC Motion System Properties}

Five programming windows were established:

1. Edit Page for motion path programming and editing.

2. On-Line Page for motion path execution.

3. Jog for $X-Y$ jog moves.

4. Joystick mode for jog moves.

5. Rectangle Generator for preprogrammed rectangular moves.

Motion path programming and editing window, shown in Figure 1, covers the creation of a motion path and/or editing of the path, step by step. The path consists of $X$ and $Y$ moves with specified clockwise or counterclockwise turns. These moves may be in Absolute or Relative mode. A rotary axis is also available which allows part rotation through $\theta$ degrees. The $X-Y$ and rotary cannot be used simultaneously with this particular hardware configuration. An $n$ second delay function is available for prepurge, post purge, laser pulse stabilization, etc. Laser power supply and shutter I/O functions are incorporated on this window as buttons, displaying Green for ON and Red for OFF. The custom software displays Prompt windows and Error messages when incorrect or conflicting information is entered.

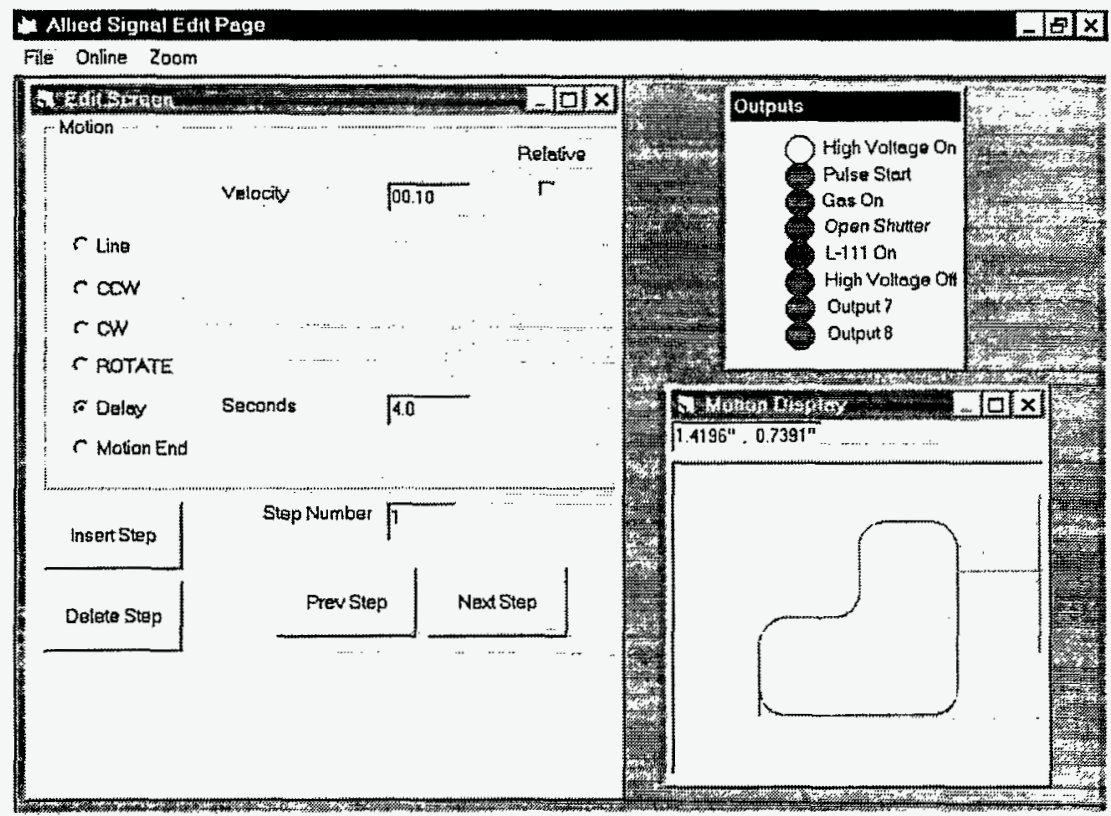

Figure 1. Edit Page Provides Path Programming and Editing 
The Motion Display window is presented within the Edit Page and On-Line windows and provides a graphic illustration of the motion path. This is an especially helpful and powerful feature of the motion system. The graphic is an adaptation of Corel $\otimes$ Visual CADD and provides a Zoom capability. True end points of the program paths can be verified by "zooming in" on a path end point or intersection. The zoomed graphic allows the operator to make sure that the numerical path inputs are correct. The Motion Display shows the path movement in real time during an actual weld.

Motion execution window, shown in Figure 2, allows the motion path to be run in one of two modes. First, the motion path can be run in a setup mode where the motion occurs without the laser being activated. The setup mode verifies motion paths and safely reveals potential path conflicts. Second, the actual run mode causes the path to be completed with the laser on and producing an active beam. This window also displays programmed distances and actual distances moved, or degrees of rotation.

Commands to Home the axis, Zero the axis, and Send Axis to Zero are available at this window.

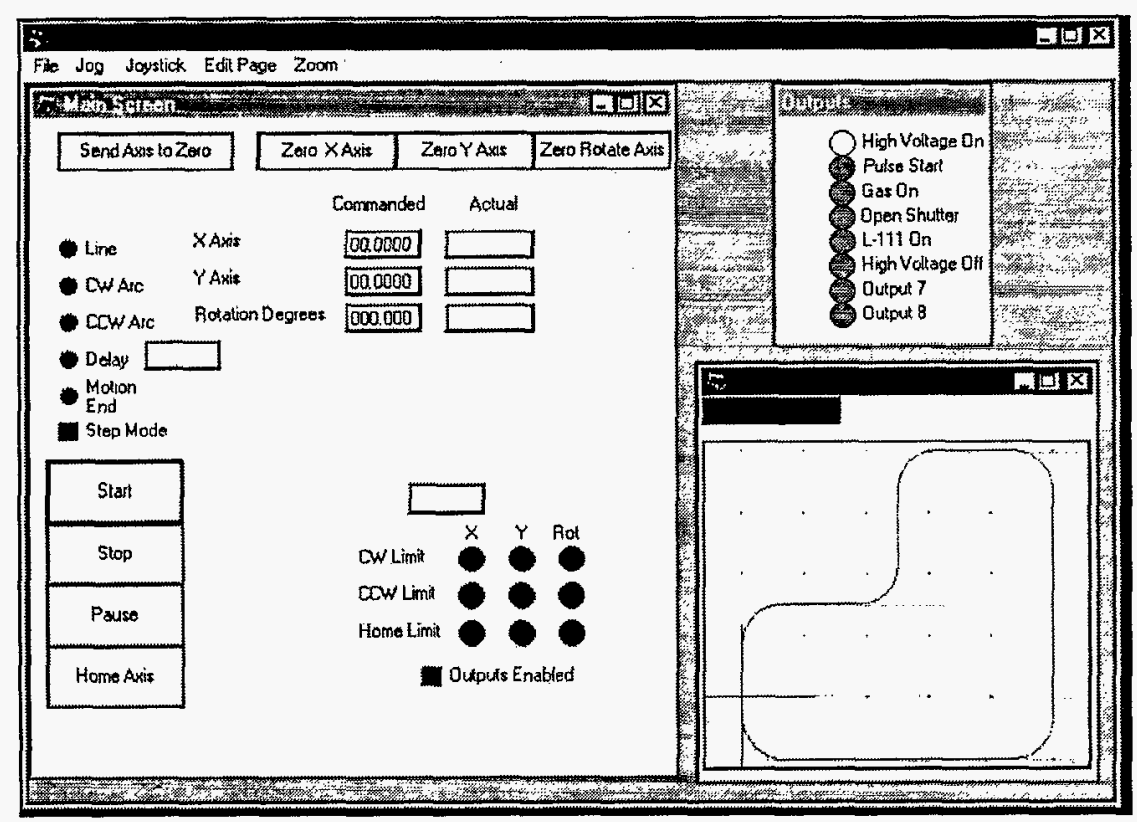

Figure 2. On-Line Page Allows Motion Execution

Figure 3 illustrates the $X-Y$ Jog feature which is used to manually move the table to a specific position by entering a numerical distance or degrees of rotation. 


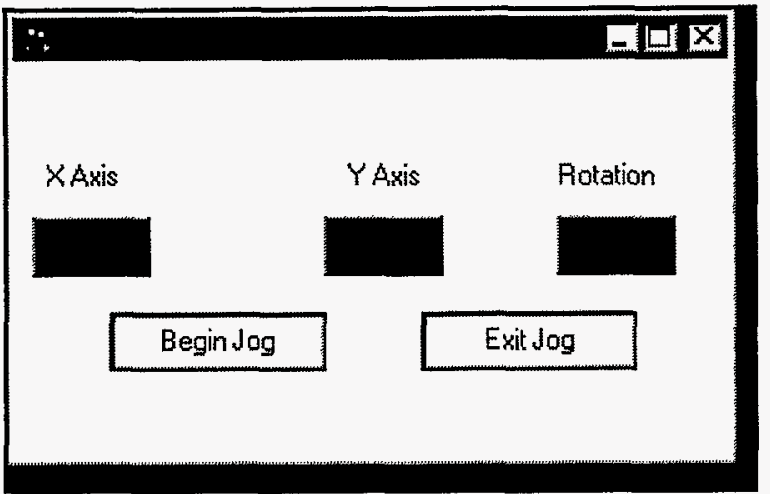

Figure 3. The Jog Window Allows Specific X-Y or Rotary Movements

The Joystick window in Figure 4 allows the $X-Y$ or rotary table to be manually controlled by a joystick. The joystick is frequently used to expedite tooling setup.

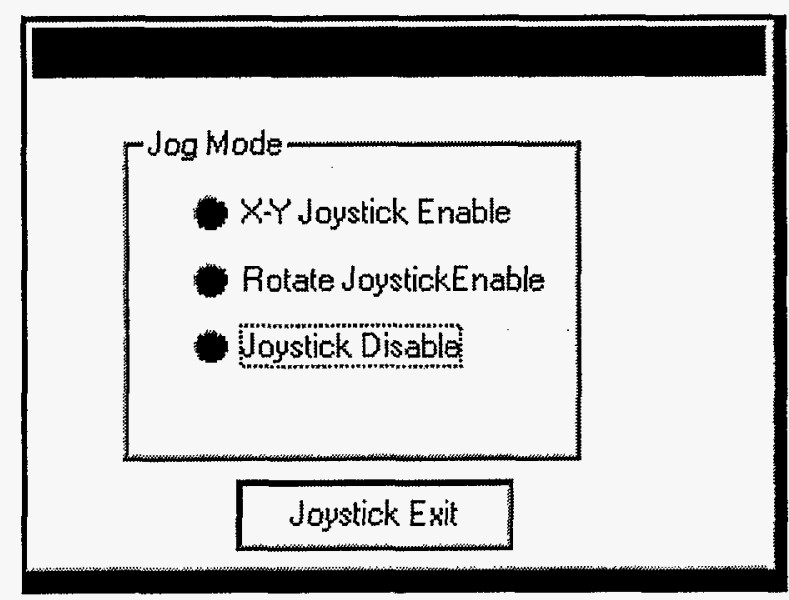

Figure 4. Joystick Control Window

The Rectangle Generator window in Figure 5 is a special case of the Edit Page window. Since the majority of the laser welding applications involve a rectangular profile, this window provides a significant programming shortcut. As shown in Figure 5, the only data required are the two sides, the corner radius, and a weld overlap, if needed. All of the standard laser $1 / O$ functions and delays are inserted in the program automatically. 


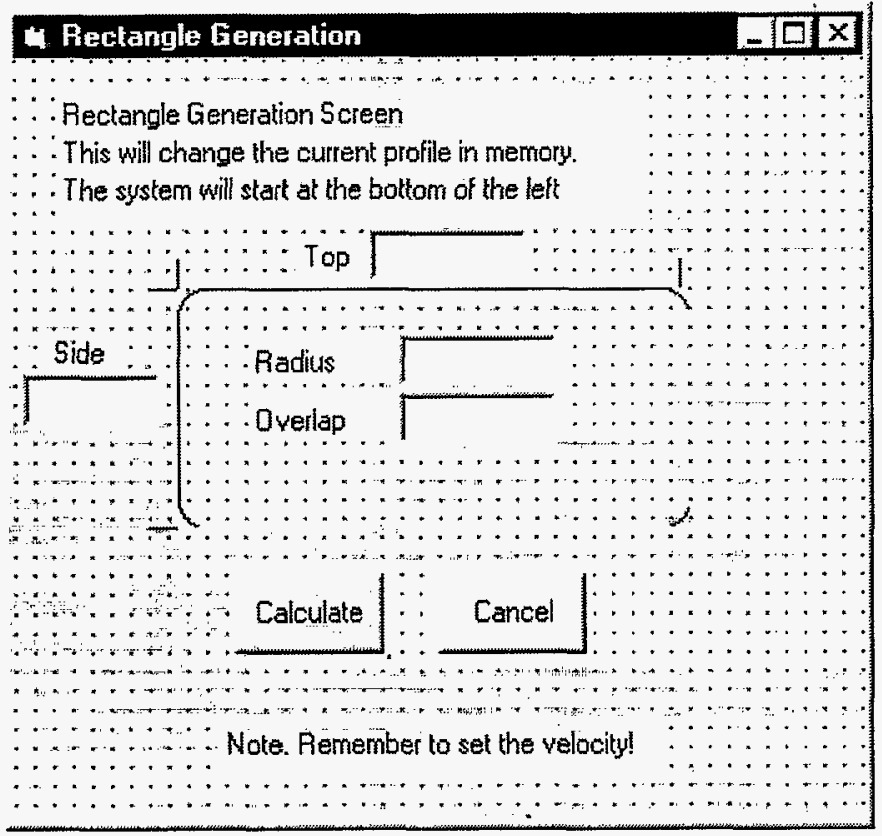

Figure 5. Rectangle Generator Window

\section{Advantages of the New Windows-Based Motion System}

Several benefits are achieved by using the Windows environment for programming and control of the laser weld motion system. All of the commands look very familiar to users experienced with other Windows applications. No previous CNC programming knowledge is required to generate, edit, and execute motion controls. The File manipulation commands are also designed like a typical Windows application. All of the motion programs are stored on the PC hard drive which allows a very generous data storage capability. Since a special protocol is required to transfer motion programs to the stepper motor electronic drives, knowledge of this protocol must be derived from the Operation Manual.

The software and hardware concepts developed on this project can be tailored to fit many different types of automation tasks. Any manufacturing operation requiring motion control, physical positioning, piece part loading/unloading, and/or //O controls could be implemented using these concepts. The bulk of the software design has been completed on this project. It is expected that only minor revisions would be required to accommodate other automation applications. 


\section{Accomplishments}

A cost-effective PC-based motion system was developed on this project and incorporated into an existing development laser welding system. The new system provides all of the features normally expected in a CNC motion system but at a fraction of the cost. The software supporting the new motion system was constructed with the user-friendly Windows type environment. FM\&T owns the software and can install it on any system desired at minimal additional cost. The use of standard stepper motor hardware provides the opportunity to use this motion system on nearly any automated motion application. 